\title{
Semigroups generated by convex combinations of several Feller generators in models of mathematical biology
}

by

\author{
ADAm Bobrowski (Katowice and Lublin) and \\ RADOSŁAW Bogucki (Warszawa)
}

Dedicated to Professor Jan Kisyński on the occasion of his 75th birthday

\begin{abstract}
Let $\mathcal{S}$ be a locally compact Hausdorff space. Let $A_{i}, i=0,1, \ldots, N$, be generators of Feller semigroups in $C_{0}(\mathcal{S})$ with related Feller processes $X_{i}=\left\{X_{i}(t), t \geq 0\right\}$ and let $\alpha_{i}, i=0, \ldots, N$, be non-negative continuous functions on $\mathcal{S}$ with $\sum_{i=0}^{N} \alpha_{i}=1$. Assume that the closure $A$ of $\sum_{k=0}^{N} \alpha_{k} A_{k}$ defined on $\bigcap_{i=0}^{N} \mathcal{D}\left(A_{i}\right)$ generates a Feller semigroup $\{T(t), t \geq 0\}$ in $C_{0}(\mathcal{S})$. A natural interpretation of a related Feller process $X=\{X(t), t \geq 0\}$ is that it evolves according to the following heuristic rules: conditional on being at a point $p \in \mathcal{S}$, with probability $\alpha_{i}(p)$, the process behaves like $X_{i}$, $i=0,1, \ldots, N$. We provide an approximation of $\{T(t), t \geq 0\}$ via a sequence of semigroups acting in the Cartesian product of $N+1$ copies of $C_{0}(\mathcal{S})$ that supports this interpretation, thus generalizing the main theorem of Bobrowski [J. Evolution Equations 7 (2007)] where the case $N=1$ is treated. The result is motivated by examples from mathematical biology involving models of gene expression, gene regulation and fish dynamics.
\end{abstract}

1. Introduction. Let $\mathcal{S}$ be be a locally compact Hausdorff space, and let $\alpha_{i}, i=0, \ldots, N$, be non-negative continuous functions on $\mathcal{S}$ with $\sum_{i=0}^{N} \alpha_{i}=1$. Furthermore, let $A_{i}, i=0, \ldots, N$, and $A$ be generators of Feller semigroups $\left\{T_{i}(t), t \geq 0\right\}$ and $\{T(t), t \geq 0\}$ in $C_{0}(\mathcal{S})$ with related Feller processes $X_{i}=\left\{X_{i}(t), t \geq 0\right\}$ and $X=\{X(t), t \geq 0\}$. Finally, suppose that $\mathcal{D}:=\bigcap_{i=0}^{N} \mathcal{D}\left(A_{i}\right)$ is a core for $A$ and that $A f=\sum_{i=0}^{N} \alpha_{i} A_{i} f$ for $f \in \mathcal{D}$. Then it is natural to expect that $X$ may be described as follows: conditional on being at $p \in \mathcal{S}, X$ behaves like $X_{i}$ with probability $\alpha_{i}(p)$.

However, neither the Trotter product formula nor the Dyson-Phillips perturbation series support such an interpretation (see [8]). (On the other hand, this interpretation agrees very well with Volkonskii's formula where,

2000 Mathematics Subject Classification: 47D07, 60J25, 60J35, 60J55.

Key words and phrases: contraction semigroup, generator, convex combination, approximation formulae, Trotter-Kato theorem, degenerate convergence, Feller process, weak convergence of processes, gene expression, gene regulation, fish dynamics. 
as in Dorroh's multiplicative perturbation theorem [15], all but one $A_{i}$ are zero; see [30, pp. 277-278] and [8].) We justify it by approximating $X$ by a sequence $\mathcal{X}_{n}, n \geq 0$, of Feller processes in the extended space $\mathcal{S}^{N+1}$ composed of $N+1$ copies $\mathcal{S} \times\{i\}, i=0,1, \ldots, N$, of $\mathcal{S}$. These processes are "mixtures" of two components. The first of these, starting on $\mathcal{S} \times\{i\}$, behaves like $X_{i}$ and never leaves $\mathcal{S} \times\{i\}$. The second is composed of independent Markov chains indexed by $p \in \mathcal{S}$ : conditional on starting from a $(p, i), i=0, \ldots, N$, it is a Markov chain with states $(p, j), j=0,1, \ldots, N$, and a certain intensity matrix depending on $n$ and $\alpha_{j}(p), j=0, \ldots, N$. This matrix is chosen so that $\left(\alpha_{i}(p)\right)_{i=0, \ldots, N}$ is its unique invariant probability measure, and the passage $n \rightarrow \infty$ corresponds to convergence of the chain to equilibrium. In the limit, points of $\mathcal{S}^{N+1}$ with the same first coordinates get glued together (the chain is at equilibrium) to form another copy of $\mathcal{S}$, and the limit process defined there may indeed be thought of as that behaving with probability $\alpha_{i}(p)$ like $X_{i}$, conditional on being at $p$.

Specifically, the processes $\left\{\mathcal{X}_{n}(t), n \geq 0\right\}$ are related to the Feller semigroups $\left\{\mathcal{T}_{n}(t), t \geq 0\right\}$ generated by the operators

$$
\mathcal{A}_{n}\left(f_{i}\right)_{i=0, \ldots, N}=\left(A_{i} f_{i}\right)_{i=0, \ldots, N}+n \mathcal{Q}\left(f_{i}\right)_{i=0, \ldots, N}, \quad f_{i} \in \mathcal{D}\left(A_{i}\right),
$$

where $C_{0}\left(\mathcal{S}^{N+1}\right)$ is identified via isometric isomorphism with the Cartesian product of $N+1$ copies of $C_{0}(\mathcal{S})$, and $\mathcal{Q}$ is a matrix whose entries are certain multiplication operators corresponding to bounded and continuous functions of $p$; for $p$ fixed, $n \mathcal{Q}(p)$ is the intensity matrix of the Markov chain described above. (In what follows, for notational convenience, we will not distinguish between a bounded, continuous function $h$ on $\mathcal{S}$ and the related multiplication operator $C_{0}(\mathcal{S}) \ni f \mapsto h f \in C_{0}(\mathcal{S})$.) In other words, although in general the $X_{i}$ 's are not deterministic, the $\mathcal{X}_{n}$ 's are somewhat similar to piecewise deterministic processes of M. H. A. Davis [12, 13, 14] in that their behavior may be described as follows: starting at the $i$ th copy of $\mathcal{S}$, they stay there, behaving like $X_{i}$ until a random time $\tau$ when they jump to the $j$ th copy of $\mathcal{S}$ to behave like $X_{j}$ there. At time $\tau$, conditional on being at $(p, i)$ they jump to $(p, j)$; the distribution of $\tau$ and the probability of choosing $j$ are determined by the matrix $\mathcal{Q}$ : its entries are the jump intensities of the processes (divided by $n$ ).

For our main Theorem 2.1, we choose

$$
\mathcal{Q}=\left(\begin{array}{ccccc}
\alpha_{0}-1 & \alpha_{1} & \alpha_{2} & \ldots & \alpha_{N} \\
\alpha_{0} & \alpha_{1}-1 & \alpha_{2} & \ldots & \alpha_{N} \\
\alpha_{0} & \alpha_{1} & \alpha_{2}-1 & \ldots & \alpha_{N} \\
\vdots & \vdots & \ddots & \ddots & \ddots \\
\alpha_{0} & \alpha_{1} & \alpha_{2} & \ldots & \alpha_{N}-1
\end{array}\right),
$$


and this choice is dictated by the following reasons. First of all $\left(\alpha_{i}(p)\right)_{i=0, \ldots, N}$ is the unique stationary measure for $\mathcal{Q}(p)$. Secondly, there are only two eigenvalues of $\mathcal{Q}(p): 0$ and 1 (in particular, they do not depend directly on $\alpha_{i}$, allowing one to avoid additional assumptions on them). These properties actually allow for a more abstract version of our result where $\alpha_{i}$ are bounded, commuting operators in a Banach space (see Theorem 2.2). At present it is unclear if the sole assumption of $\left(\alpha_{i}(p)\right)_{i=0, \ldots, N}$ being the unique stationary measure for $\mathcal{Q}(p)$ suffices for convergence of $\left\{\mathcal{I}_{n}(t), t \geq 0\right\}$ to $\{T(t), t \geq 0\}$.

Similar "averaging" procedures are known in mathematical physics (see e.g. bibliography given in [2]). In the context of stochastic processes, in [33] the case where $A_{n}$ are generators of motions along integral curves of ordinary differential equations is studied. For the case where $A_{n}$ are diffusion operators, see the literature on so-called fast switching diffusions, e.g. [21, 40, 41].

2. Main result. Let $\mathbb{X}$ be a Banach space; its elements will be denoted $x, y, z$ etc. with possible subscripts. Also, let $N \geq 1$ be fixed and let $\mathbb{Y}$ be the Cartesian product of $N+1$ copies of $\mathbb{X}$. Elements of this space will be denoted by $\mathbf{x}, \mathbf{y}, \mathbf{z},\left(x_{0}, x_{1}, \ldots, x_{N}\right),\left(x_{i}\right)_{i=0, \ldots, N}$, etc. We will also write $[x]$ for the element $(x, \ldots, x)$, where $x \in \mathbb{X}$. Operators in $\mathbb{X}$ will be denoted $A, B$ etc. and those in $\mathbb{Y}$ by $\mathcal{A}, \mathcal{B}$ etc. with possible subscripts (the only exception to this rule are the special operators $\alpha_{i}$ to be described below).

Elements of $C_{0}(\mathcal{S})$, the space of continuous functions vanishing at infinity, defined on $\mathcal{S}$, a locally compact Hausdorff space, will be denoted by $f, g, h$, etc. The Cartesian product of $N+1$ copies of this space will be denoted by $\mathbb{B}$ and its elements will be denoted analogously to elements of $\mathbb{Y}$ described above.

We denote $\alpha_{i}, i=0, \ldots, N$, commuting operators in $\mathbb{X}$ such that $\sum_{i=0}^{N} \alpha_{i}$ $=I_{\mathbb{X}}$. In the context of $C_{0}(\mathcal{S}), \alpha_{i}$ 's are always non-negative, continuous functions on $\mathcal{S}$ with the above property. We set

$$
\operatorname{conv} \mathbf{x}=\operatorname{conv}\left(x_{i}\right)_{i=0, \ldots, N}=\sum_{i=0}^{N} \alpha_{i} x_{i} .
$$

Our main theorem is:

Theorem 2.1. Let $A_{i}, i=0, \ldots, N$, and $A$ be the generators of Feller semigroups $\left\{T_{i}(t), t \geq 0\right\}$ and $\{T(t), t \geq 0\}$ in $C_{0}(\mathcal{S})$. Also suppose that $A f=\sum_{i=0}^{N} \alpha_{i} A_{i} f=\operatorname{conv}\left(A_{i} f\right)_{i=0, \ldots, N}$ for $f \in \mathcal{D}:=\bigcap_{i=0}^{N} \mathcal{D}\left(A_{i}\right)$ and that $\mathcal{D}$ is a core for $A$. Finally, let $\mathcal{A}_{n}, n \geq 0$, with $\mathcal{D}\left(\mathcal{A}_{n}\right)=\mathcal{D}\left(A_{0}\right) \times \mathcal{D}\left(A_{1}\right) \times$ $\cdots \times \mathcal{D}\left(A_{N}\right) \subset \mathbb{Y}$ be given by (1) and (2) so that

$$
\mathcal{Q} \mathbf{f}=\mathcal{Q}\left(f_{i}\right)_{i=0, \ldots, N}=\left(\operatorname{conv} \mathbf{f}-f_{i}\right)_{i=0, \ldots, N}
$$


Then each $\mathcal{A}_{n}$ generates a contraction semigroup $\left\{\mathcal{T}_{n}(t), t \geq 0\right\}$ in $\mathbb{B}$ and

$$
\lim _{n \rightarrow \infty} \mathcal{T}_{n}(t) \mathbf{f}=[T(t) \operatorname{conv} \mathbf{f}], \quad t>0, \mathbf{f} \in \mathbb{B} .
$$

In fact, we prove a more general, abstract version of Theorem 2.1:

Theorem 2.2. Let $A_{i}, i=0, \ldots, N$, and $A$ be the generators of semigroups $\left\{T_{i}(t), t \geq 0\right\}$ and $\{T(t), t \geq 0\}$ in a Banach space $\mathbb{X}$. Also suppose that $A x=\operatorname{conv}\left(A_{i} x\right)_{i=0, \ldots, N}$ for $x \in \mathcal{D}:=\bigcap_{i=0}^{N} \mathcal{D}\left(A_{i}\right)$ and that $\mathcal{D}$ is a core for $A$. Finally, let $\mathcal{A}_{n}, n \geq 0$, with $\mathcal{D}\left(\mathcal{A}_{n}\right)=\mathcal{D}\left(A_{0}\right) \times \mathcal{D}\left(A_{1}\right) \times \cdots \times \mathcal{D}\left(A_{N}\right) \subset$ $\mathbb{Y}$ be given by

$$
\begin{aligned}
\mathcal{A}_{n}\left(x_{i}\right)_{i=0, \ldots, N}=\left(A_{i} x_{i}\right)_{i=0, \ldots, N}+n \mathcal{Q}\left(x_{i}\right)_{i=0, \ldots, N} & \\
& x_{i} \in \mathcal{D}\left(A_{i}\right), i=0, \ldots, N,
\end{aligned}
$$

where

$$
\mathcal{Q}\left(x_{i}\right)_{i=0, \ldots, N}=\left(\operatorname{conv} \mathbf{x}-x_{i}\right)_{i=0, \ldots, N},
$$

and suppose that the $\mathcal{A}_{n}$ 's generate equibounded semigroups $\left\{\mathcal{T}_{n}(t), t \geq 0\right\}$ in $\mathbb{Y}$. Then

$$
\lim _{n \rightarrow \infty} \mathcal{T}_{n}(t) \mathbf{x}=[T(t) \operatorname{conv} \mathbf{x}], \quad t>0, \mathbf{x} \in \mathbb{X} .
$$

Our theorem involves "double convex combination" in that in the limit we have the semigroup generated by a convex combination of generators, acting on a convex combination of initial conditions.

Theorem 2.1 is a special case of Theorem 2.2. Indeed, under the assumptions of Theorem 2.1, $\mathcal{A}_{0}$ is the generator of the semigroup $\left\{\mathcal{T}_{0}(t), t \geq 0\right\}$ given by $\mathcal{T}_{0}(t)\left(f_{i}\right)_{i=0, \ldots, N}=\left(T_{i}(t) f_{i}\right)_{i=0, \ldots, N}$. Moreover, $\mathcal{Q}$ is a bounded operator. Hence, by the Phillips perturbation theorem $\mathcal{A}_{n}$ generates a strongly continuous semigroup. Moreover, $\mathcal{Q}$ is also the generator of a contraction semigroup. Hence, by the Trotter product formula, so is $\mathcal{A}_{n}=\mathcal{A}_{0}+n \mathcal{Q}$, and all the assumptions of Theorem 2.2 are satisfied.

Both theorems are examples of degenerate convergence of semigroups (see $[4,5,7,8,9]$ ). In particular, for $\mathbf{x}=[x], x \in \mathbb{X},(7)$ is true for $t=0$ as well and the limit is almost uniform in $t \in[0, \infty)$ while for the remaining $\mathbf{x}$ it is almost uniform in $t \in(0, \infty)$.

Although the following example is inspired by the "Single gene, no feedback" section of [22] (see in particular equations (4), (5) and (21) there), it is meant to be merely an illustration of our Theorem 2.2. In particular, we do not claim it represents any biologically meaningful reality.

EXAmple (Poisson processes with changing colors). Let $\alpha_{i}=\left(\alpha_{i, p}\right)_{p \geq 1}$, $i=0, \ldots, N$, be positive, convergent sequences with $\sum_{i=0}^{N} \alpha_{i}=1$, and $c_{i}, i=0, \ldots, N$, be positive constants. (In other words, we are in $\mathcal{S}=\mathbb{N}$.) The state space of the sequence $\left\{\mathcal{X}_{n}(t), t \geq 0\right\}, n \geq 1$, of processes to be 
described is $\mathbb{N} \times\{0, \ldots, N\}$ with the first coordinate denoting the state of a regular Poisson process, and the second its changing color. The process $\mathcal{X}_{n}(t), t \geq 0$, starting at $(p, i)$ waits there for an exponential time with parameter $c_{i}+n\left(1-\alpha_{i}(p)\right)$ to jump, with probability $c_{i} /\left(c_{i}+n\left(1-\alpha_{i}(p)\right)\right)$ to $(p+1, i)$ or, with probability $n \alpha_{j}(p) /\left(c_{i}+n\left(1-\alpha_{i}(p)\right)\right)$, to $(p, j), j \neq i$. Equivalently, $\mathcal{X}_{n}(t), t \geq 0$, is a "mixture" of two processes: the first of them, at the $i$ th copy of $\mathbb{N}$, is a Poisson process with intensity $c_{i}$; the other is composed of independent $p$-indexed color change Markov chains with values in $\{(p, 0), \ldots,(p, N)\}$ and intensity matrix $n \mathcal{Q}$ where $\mathcal{Q}$ is given by $(2)$ with $\alpha_{i}=\alpha_{i}(p)$. This mixture is a Feller process and its generator (after suitable identification of isomorphic objects) is

$$
\mathcal{A}_{n}\left(x_{i}\right)_{i=0, \ldots, N}=\left(c_{i} P x_{i}\right)_{i=0, \ldots, N}+n Q\left(x_{i}\right)_{i=0, \ldots, N},
$$

where $\left(x_{i}\right)_{i=0, \ldots, N}$ belongs to the space $\mathbb{B}$, the Cartesian product of $N+1$ copies of $c_{0}=c_{0}(\mathbb{N})$, and $P$ is the operator in $c_{0}$ given by $P\left(\xi_{p}\right)_{p \geq 1}=$ $\left(\xi_{p+1}-\xi_{p}\right)_{p \geq 1}$.

For large $n$, the probability of Poisson-type transition $(p, i) \mapsto(p+1, i)$ is small (provided $\alpha_{i}(p) \neq 1$ ), while transitions $(p, i) \mapsto(p, j)$ occur relatively often. As a result, before a Poisson-type transition occurs, the color change process reaches its equilibrium, so that we are at $(p, j)$ with probability $\alpha_{j}(p)$. Hence, in the limit, the intensity of Poisson-type transition becomes state dependent and equals $\sum_{i=0}^{N} c_{i} \alpha_{i}(p)$. The same is true in the case where $\alpha_{i}(p)=1$, when the process, after waiting for an exponential time with parameter $c_{i}$ jumps right away to $(p+1, i)$. In other words, the processes $\left\{\mathcal{X}_{n}(t), t \geq 0\right\}, n \geq 1$, approximate the process in $\mathbb{N}$ with generator

$$
A\left(\xi_{p}\right)_{p \geq 1}=\left(\sum_{i=0}^{N} c_{i} \alpha_{i}(p) \xi_{p+1}-\xi_{p}\right)_{p \geq 1},
$$

i.e. a pure birth process with intensities averaged over equilibrium states. Theorem 2.2 makes these intuitions precise.

3. Proof of Theorem 2.2. Let $\mathcal{P}: \mathbb{Y} \rightarrow \mathbb{Y}$ be given by $\mathcal{P} \mathbf{x}=[\operatorname{conv} \mathbf{x}]$. Then $\mathcal{P}=\mathcal{Q}+\mathcal{I}$, where $\mathcal{I}$ is the identity on $\mathbb{Y}, \mathcal{P}$ is idempotent $\left(\mathcal{P}^{2}=\mathcal{P}\right)$ and $\mathbb{Y}$ is a direct sum of two subspaces

$$
\begin{aligned}
& \mathbb{Y}_{0}=\text { Range } \mathcal{P}=\operatorname{Ker} \mathcal{Q}=\{[x]: x \in \mathbb{X}\}, \\
& \mathbb{Y}_{1}=\operatorname{Ker} \mathcal{P}=\text { Range } \mathcal{Q}=\{\mathbf{x} \in \mathbb{Y}: \operatorname{conv} \mathbf{x}=0\} .
\end{aligned}
$$

3.1. Proof of $\mathcal{D}\left(\mathcal{A}_{\mathrm{ex}}\right) \subset \mathbb{Y}_{0}$. Let $\mathcal{A}_{\mathrm{ex}}$ be the extended limit of the generators $\mathcal{A}_{n}$, which is a relation in $\mathbb{Y} \times \mathbb{Y}$ (see $\left.[6,17,23,36]\right)$. An element $\mathbf{x}$ belongs to $\mathcal{D}\left(\mathcal{A}_{\text {ex }}\right)$ iff there exists a sequence $\mathbf{x}_{n} \in \mathcal{D}\left(\mathcal{A}_{n}\right), n \geq 1$, such 
that $\lim _{n \rightarrow \infty} \mathbf{x}_{n}=\mathbf{x}$ and the $\operatorname{limit}_{\lim } \rightarrow \infty \mathcal{A}_{n} \mathbf{x}_{n}$ exists. In what follows, by $\mathcal{A}_{\text {ex }} \mathbf{x}=\mathbf{z}$ we mean $(\mathbf{x}, \mathbf{z}) \in \mathcal{A}_{\text {ex }}$. If $\lim _{n \rightarrow \infty}\left(\mathcal{A}_{0} \mathbf{x}_{n}+n \mathcal{Q} \mathbf{x}_{n}\right)=\mathbf{y}$ and $\lim _{n \rightarrow \infty} \mathbf{x}_{n}=\mathbf{x}$, then $\lim _{n \rightarrow \infty}\left[\mathcal{A}_{0} n^{-1} \mathbf{x}_{n}+\mathcal{Q} \mathbf{x}_{n}\right]=0$ and $\mathcal{Q} \mathbf{x}_{n}$ converges. Hence, $\mathcal{A}_{0} n^{-1} \mathbf{x}_{n}$ converges, whence, as $\lim _{n \rightarrow \infty} n^{-1} \mathbf{x}_{n}=0$, it converges to zero, $\mathcal{A}_{0}$ being closed. Thus, $\lim _{n \rightarrow \infty} \mathcal{Q} \mathbf{x}_{n}=\mathcal{Q} \mathbf{x}=0$, proving that $\mathbf{x} \in \operatorname{Ker} \mathcal{Q}$.

3.2. Proof of $\mathcal{A}_{\mathrm{ex}}[x]=\mathcal{A}_{0}[x]+\mathcal{Q} \mathbf{z}$ for $x \in \mathcal{D}$ and $\mathbf{z} \in \mathbb{Y}$. Given $x \in \mathcal{D}$ and $\mathbf{z} \in \mathbb{Y}$, we define $\mathbf{z}_{n}=\sqrt{n}\left(\sqrt{n}-\mathcal{A}_{0}\right)^{-1} \mathbf{z}$, and $\mathbf{y}_{n}=[x]+n^{-1} \mathbf{z}_{n}, n \geq 1$. Then $\mathbf{z}_{n} \in \mathcal{D}\left(\mathcal{A}_{0}\right)$, and $\lim _{n \rightarrow \infty} \mathbf{z}_{n}=\mathbf{z}$. Moreover, by the resolvent equation,

$$
\left\|\mathcal{A}_{0} \mathbf{z}_{n}\right\|=\sqrt{n}\left\|\sqrt{n}\left(\sqrt{n}-\mathcal{A}_{0}\right)^{-1} \mathbf{z}-\mathbf{z}\right\| \leq \sqrt{n}(M+1)\|z\|,
$$

where $M$ is a constant such that $\left\|\mathcal{T}_{0}(t)\right\| \leq M$ for $t \geq 0$. Therefore, $\mathbf{y}_{n} \in$ $\mathcal{D}\left(\mathcal{A}_{n}\right), \lim _{n \rightarrow \infty} \mathbf{y}_{n}=[x]$ and $\mathcal{A}_{n} \mathbf{y}_{n}=\mathcal{A}_{0}[x]+\mathcal{A}_{0} n^{-1} \mathbf{z}_{n}+\mathcal{Q} \mathbf{z}_{n}$ tends to $\mathcal{A}_{0}[x]+\mathcal{Q} \mathbf{z}$. This proves that $[x]$ is in the domain of the extended limit of $\mathcal{A}_{n}$ and $\mathcal{A}_{0}[x]+\mathcal{Q} \mathbf{z}$ is one of the possible values of $\mathcal{A}$ at $[x]$.

3.3. Proof of $\lim _{n \rightarrow \infty} \mathcal{T}_{n}(t)[x]=[T(t) x], x \in \mathbb{X}$. Fix $\lambda>0$. For $x \in \mathcal{D}$ and $\mathbf{w} \in \mathbb{Y}$, taking $\mathbf{z}=\mathcal{A}_{0}[x]-\mathbf{w}$ in Subsection 3.2 we see that $[\lambda x-A x]+\mathcal{Q} \mathbf{w}$ is a possible value of $\lambda \mathcal{I}-\mathcal{A}_{\mathrm{ex}}$ at $[x]$. Since $\mathcal{D}$ is a core for $A$, and $A$ is the generator of a semigroup, the range of $\lambda I-A$ as restricted to $\mathcal{D}$ is dense in $\mathbb{X}$. Hence the range of $\lambda \mathcal{I}-\mathcal{A}_{\mathrm{ex}}$ is dense in $\mathbb{Y}$, or, which is the same $[6,7,17]$, the resolvents of $\left\{\mathcal{T}_{n}(t), t \geq 0\right\}$ converge strongly on $\mathbb{Y}$. By Subsection 3.1, the range of the limit pseudo-resolvent, being equal to $\mathcal{D}\left(\mathcal{A}_{\mathrm{ex}}\right)$, is contained in $\mathbb{Y}_{0}$. On the other hand, by Subsection 3.2, this range contains $\{\mathbf{x} \in \mathbb{Y}: \mathbf{x}=[x], x \in \mathcal{D}\}$ and so its closure equals $\mathbb{Y}_{0}$, for $\mathcal{D}$ is dense in $\mathbb{X}$.

Thus, by the Sova-Kurtz version of the Trotter-Kato theorem $[6,17$, 23, 36], $\lim _{n \rightarrow \infty} \mathcal{T}_{n}(t)[x]$ exists for $x \in \mathbb{X}$, almost uniformly in $t \in[0, \infty)$, defining a semigroup on $\mathbb{Y}_{0}$, and the generator of this limit semigroup is the part $\mathcal{A}_{p}$ of $\mathcal{A}_{\mathrm{ex}}$ in $\mathbb{Y}_{0}$. Since $\mathcal{A}_{p}$ is single-valued and $\mathcal{D}$ is a core for $A$, and one of possible values of $\mathcal{A}_{p}$ at $[x]$ is $[A x]$, we see that the limit semigroup is the isometrically isomorphic copy of $\{T(t), t \geq 0\}$ in $\mathbb{Y}_{0}$, as desired.

3.4. Proof of $\lim _{n \rightarrow \infty} \mathcal{T}_{n}(t) \mathbf{x}=0, \mathbf{x} \in \mathbb{Y}_{1}$. The idea of the proof is to compare the sequence $\left\{\mathcal{T}_{n}(t), t \geq 0\right\}, n \geq 0$, with a sequence of semigroups $\left\{\mathcal{U}_{n}(t), t \geq 0\right\}, n \geq 0$, and check convergence of the latter, together with convergence of their difference on $\mathbb{Y}_{1}$. Let operators $\mathcal{G}_{n}$ with domains $\mathcal{D}\left(\mathcal{G}_{n}\right)=\mathcal{D}\left(\mathcal{A}_{n}\right)$ be given by $\mathcal{G}_{n}=\mathcal{A}_{0}-n \mathcal{I}$; each $\mathcal{G}_{n}$ generates a strongly continuous semigroup given by $\mathcal{U}_{n}(t)=e^{-n t} \mathcal{T}_{0}(t)$. We have $\left(\mathcal{G}_{n}-\mathcal{A}_{n}\right) \mathbf{x}=-n \mathcal{P} \mathbf{x}$ for $\mathbf{x} \in \mathcal{D}\left(\mathcal{A}_{n}\right)$, and $\lim _{n \rightarrow \infty}\left\|\mathcal{U}_{n}(t)\right\|=0$ for $t>0$. Also, for $\mathbf{x} \in \mathcal{D}\left(\mathcal{A}_{n}\right)=$ $\mathcal{D}\left(\mathcal{G}_{n}\right)$ 


$$
\begin{aligned}
\mathcal{U}_{n}(t) \mathbf{x}-\mathcal{T}_{n}(t) \mathbf{x} & =\left.\mathcal{T}_{n}(t-s) \mathcal{U}_{n}(s) \mathbf{x}\right|_{s=0} ^{s=t}=\int_{0}^{t} \frac{d}{d s}\left(\mathcal{T}_{n}(t-s) \mathcal{U}_{n}(s)\right) \mathbf{x} d s \\
& =\int_{0}^{t} \mathcal{T}_{n}(t-s)\left(\mathcal{G}_{n}-\mathcal{A}_{n}\right) \mathcal{U}_{n}(s) \mathbf{x} d s \\
& =-n \int_{0}^{t} e^{-n s} \mathcal{T}_{n}(t-s) \mathcal{P} \mathcal{T}_{0}(s) \mathbf{x} d s .
\end{aligned}
$$

Since $\mathcal{D}\left(\mathcal{A}_{n}\right)$ is dense in $\mathbb{Y}$ and all the operators involved in the left-most and right-most expressions above are bounded, these expressions are equal for all $\mathbf{x} \in \mathbb{Y}$. Therefore, $\left\|\mathcal{U}_{n}(t) \mathbf{x}-\mathcal{T}_{n}(t) \mathbf{x}\right\| \leq$ const $\cdot \int_{0}^{t} n e^{-n s}\left\|\mathcal{P} \mathcal{T}_{0}(s) \mathbf{x}\right\| d s$ Now, $\lim _{s \rightarrow 0} \mathcal{P} \mathcal{T}_{0}(s) \mathbf{x}=\mathcal{P} \mathbf{x}=0$ for $\mathbf{x} \in \mathbb{Y}_{1}$. Thus, the result follows by the well-known fact that the measures on $[0, \infty)$ with densities $s \mapsto n e^{-n s}$ converge weakly, as $n \rightarrow \infty$, to the Dirac measure at 0 .

3.5. Proof of (7). We have

$$
\lim _{n \rightarrow \infty} \mathcal{T}_{n}(t) \mathbf{x}=\lim _{n \rightarrow \infty} \mathcal{T}_{n}(t)(\mathbf{x}-\mathcal{P} \mathbf{x})+\lim _{n \rightarrow \infty} \mathcal{T}_{n}(t) \mathcal{P} \mathbf{x}=0+[T(t) \operatorname{conv} \mathbf{x}]
$$

\section{Examples of convex combinations of Feller generators in applied sciences}

4.1. A binomial type convex combination in a model of gene expression. Gene expression is the process in which a gene's DNA sequence is converted into the structures and functions of a cell (most often: proteins or RNA). The system of differential equations

$$
\begin{aligned}
& \frac{d x}{d t}=\gamma-x, \\
& \frac{d y}{d t}=r(x-y),
\end{aligned}
$$

where $r>0$ is a given constant and $\gamma$ is a stochastic process with values in $\{0,1, \ldots, N\}$ to be described in more detail below, was used as a model of gene expression by Lipniacki et al. in $[10,19,25,26,29]$. The idea of the model is as follows. The existence of a particular protein in the cell is an indicator of the existence and activity of a gene there; there are $N$ copies of the gene and $\gamma(t)$ of them are active at time $t$. The protein level $y$ is regulated by the number of active gene copies via the level $x$ of mRNA (messenger RNA): if all the gene copies are inactive $(\gamma=0), x$ decreases exponentially due to the natural process of decay, and the protein level $y$ follows $x$ with efficiency $r$; at activation of one of the gene copies $(\gamma \geq 1)$, the mRNA strives to reach level $x=\gamma$, and $y$ follows $x$ in a similar fashion as before. On the other hand, a copy of the gene is activated or deactivated with intensities $a q_{\mathrm{a}}(x, y)$ and $b q_{\mathrm{d}}(x, y)$, where $a$ and $b$ are positive constants, 
and $q_{\mathrm{a}}$ and $q_{\mathrm{d}}$ are positive functions on the square $\mathcal{S}=[0, N]^{2}$; the subscript "a" stands for "activation", and "d" for "deactivation". Since the copies are activated and deactivated independently, $\gamma$ is a $\{0, \ldots, N\}$-valued process such that, given $\gamma=i$, its intensity of increase by 1 is $(N-i) a q_{\mathrm{a}}(x, y)$ and intensity of decrease by 1 is $i b q_{\mathrm{d}}(x, y)$. Between activation/deactivation jumps, the process is deterministic. The triple $(x(t), y(t), \gamma(t)), t \geq 0$, is a Markov process, a particular case of piecewise deterministic processes of M. H. A. Davis [12, 13, 14] (see [10] for additional information).

If $a$ and $b$ tend to infinity in such a way that $a / b$ tends to a positive constant $c$, the jumps occur infinitely often, and it may be expected that $\gamma$ reaches statistical equilibrium and may be replaced in (9) by its conditional expected value (given $x$ and $y$ ):

$$
\begin{aligned}
& \frac{d x}{d t}=N \alpha(x, y)-x, \\
& \frac{d y}{d t}=r(x-y),
\end{aligned}
$$

where $\alpha=c q_{\mathrm{a}} /\left(c q_{\mathrm{a}}+q_{\mathrm{d}}\right)$. This passage to the limit corresponds to the classical case of frequent transitions (see [1,35], cf. [11, 22] and [37]).

To see that this result involves a semigroup generated by a convex combination of Feller generators, let $\mathbb{Y}$ be the Cartesian product of $N+1$ copies of $C=C(\mathcal{S})$, and $C^{1}$ be the space of continuously differentiable functions on $\mathcal{S}$. Also, let

$$
\mathcal{A}_{a, b} \mathbf{f}=\left(A_{i} f_{i}\right)_{i=0, \ldots, N}+\mathcal{Q} \mathbf{f}, \quad f_{i} \in \mathcal{D}\left(A_{i}\right), i=0, \ldots, N,
$$

where $A_{i}, i \in\{0, \ldots, N\}$, is the closure in $C$ of the map $C^{1} \ni f \mapsto g \in C$ where

$$
g(x, y)=A_{i} f(x, y)=(i-x) \frac{\partial f}{\partial x}(x, y)+r(x-y) \frac{\partial f}{\partial y}(x, y)
$$

and

$$
\begin{aligned}
& \mathcal{Q}\left(f_{i}\right)_{i=0, \ldots, N} \\
& \quad=\left(i b q_{\mathrm{d}} f_{i-1}+\left(-(N-i) a q_{\mathrm{a}}-i b q_{\mathrm{d}}\right) f_{i}+(N-i) a q_{\mathrm{a}} f_{i+1}\right)_{i=0, \ldots, N},
\end{aligned}
$$

with $f_{-1}:=f_{N+1}:=0$. The operator (11) is the generator of a semigroup $\left\{T_{a, b}(t), t \geq 0\right\}$ in $\mathbb{Y}$, related to (9), and the hypothesis may be expressed as

$$
\lim _{a, b \rightarrow \infty, a / b \rightarrow c>0} T_{a, b}(t) \mathbf{f}=[T(t) \operatorname{conv} \mathbf{f}], \quad t>0,
$$

where $\operatorname{conv}\left(f_{i}\right)_{i=0, \ldots, N}=\sum_{i=0}^{N}\left(\begin{array}{c}N \\ i\end{array}\right) \alpha^{i} \beta^{N-i} f_{i}, \beta=1-\alpha$, and the semigroup $\{T(t), t \geq 0\}$ in $C$ is generated by the closure $A$ of the map $C^{1} \ni f \mapsto g \in C$ where

$$
g(x, y)=A f(x, y)=(N \alpha(x, y)-x) \frac{\partial f}{\partial x}(x, y)+r(x-y) \frac{\partial f}{\partial y}(x, y) .
$$


For $N=1$, this hypothesis is proved in [7]; the general proof will be published elsewhere.

Now, as announced, $A$ is a convex combination of $A_{i} \mathrm{~s}$ :

$$
A f=\sum_{i=0}^{N}\left(\begin{array}{c}
N \\
i
\end{array}\right) \alpha^{i} \beta^{N-i} A_{i} f, \quad f \in C^{1} \text { (with } C^{1} \text { being a core for } A \text { ) }
$$

since $\sum_{i=0}^{N} i\left(\begin{array}{c}N \\ i\end{array}\right) \alpha^{i} \beta^{N-i}$, the expectation of the binomial distribution, equals $N \alpha$. This result is intuitively clear: the binomial distribution is the unique invariant probability measure for the chain governed by $\mathcal{Q}$ given in (12). It must be, since all gene copies are activated/deactivated independently with probability of a particular gene being active equal to $\alpha=\alpha(x, y)$.

4.2. Fast operator fluctuations in a model of gene regulation. One of the ways cells regulate gene expression is by binding so-called regulatory proteins (either activating or repressing) to a regulatory sequence in the gene. This mechanism is particularly well developed in prokaryote where the regulatory sequence is termed the operator. The system of equations

$$
\frac{d x_{i}(t)}{d t}=F_{i}(\gamma(t))-x_{i}(t), \quad i=1,2,
$$

where $F_{1}(0)=F_{1}(1)=F_{2}(0)=F_{2}(2)=1$ and $F_{1}(2)=F_{2}(1)=0$, describes one of possible realizations of such a biological situation (see [22, section "Regulated systems II: mutual repressors"]). Here, two genes share the same operator and their products (proteins) act as mutual repressors: $x_{i} \in[0,1]$, $i=1,2$ is the level of the $i$ th protein, and $\gamma \in\{0,1,2\}$ denotes the state of the operator, 0 referring to the unoccupied state, 1 to being occupied by a dimer of the first protein, and 2 to being occupied by a dimer of the second one. If none of the proteins is bound to the operator $(\gamma=0)$, both are being produced at a common, constant rate. $\gamma(t)$ jumps from 0 to 1 with intensity $\kappa x_{1}^{2}$ and from 0 to 2 with intensity $\kappa x_{2}^{2}$ with $\kappa$ a given constant, since proteins may bind to the operator merely in the dimer form, and dimers are assumed to be formed and then bound to the operator at the rate proportional to the square of monomer level. When one of the proteins is bound to the operator, production of the other is repressed, and the existing molecules gradually degrade. However, with constant intensity, dimers leave the operator, thus triggering production of the protein previously repressed: $\gamma$ jumps from states 1 and 2 to 0 with intensity $\kappa b$ where $b$ is a certain positive constant. Hence, the cycle is repeated with possible change of roles between proteins.

The Kolmogorov equation for the resulting process $\left(x_{1}(t), x_{2}(t), \gamma(t)\right)$, $t \geq 0$, is (cf. [22, eqs. (C1)-(C3) in Appendix C]): 


$$
\begin{aligned}
& \frac{\partial f_{0}}{\partial t}=\left(1-x_{1}\right) \frac{\partial f_{0}}{\partial x_{1}}+\left(1-x_{2}\right) \frac{\partial f_{0}}{\partial x_{2}}-\kappa\left(x_{1}^{2}+x_{2}^{2}\right) f_{0}+\kappa x_{1}^{2} f_{1}+\kappa x_{2}^{2} f_{2}, \\
& \frac{\partial f_{1}}{\partial t}=\left(1-x_{1}\right) \frac{\partial f_{1}}{\partial x_{1}}+\left(-x_{2}\right) \frac{\partial f_{1}}{\partial x_{2}}+\kappa b f_{0}-\kappa b f_{1}, \\
& \frac{\partial f_{2}}{\partial t}=\left(-x_{1}\right) \frac{\partial f_{2}}{\partial x_{1}}+\left(1-x_{2}\right) \frac{\partial f_{2}}{\partial x_{2}}+\kappa b f_{0}-\kappa b f_{2},
\end{aligned}
$$

where for fixed $t, f_{0}, f_{1}, f_{2}$ belong to $C\left([0,1]^{2}\right)$. In other words, we are dealing with equation (1) with $\mathcal{S}=[0,1]^{2}, N=2$, with integer $n$ replaced by real $\kappa$,

$$
Q\left(f_{i}\right)_{i=0,1,2}=\left(-\left(x_{1}^{2}+x_{2}^{2}\right) f_{0}+x_{1}^{2} f_{1}+x_{2}^{2} f_{2}, b f_{0}-b f_{1}, b f_{0}-b f_{2}\right),
$$

and

$$
A_{i} f\left(x_{1}, x_{2}\right)=\left(F_{1}(i)-x_{1}\right) \frac{\partial f}{\partial x_{1}}+\left(F_{2}(i)-x_{2}\right) \frac{\partial f}{\partial x_{2}}, i=0,1,2, f \in C^{1} ;
$$

$C^{1}$, the subspace of continuously differentiable functions, is a core for all of these three operators.

The constant $\kappa$ describes the speed at which the operator fluctuates (see [22, eqs. (2) and (33)]). Hence, as $\kappa \rightarrow \infty$, the operator reaches its (conditional) statistical equilibrium with probabilities of being at 0,1 and 2 equal to $\alpha_{0}=b w^{-1}, \alpha_{1}=x_{1}^{2} w^{-1}$ and $\alpha_{2}=x_{2}^{2} w^{-1}$, respectively, where $w=b+x_{1}^{2}+x_{2}^{2}$. Hence, (13) becomes

$$
\frac{d x_{i}(t)}{d t}=\frac{b+x_{i}^{2}}{b+x_{1}^{2}+x_{2}^{2}}-x_{i}(t), \quad i=1,2 .
$$

a reproduction of (46) and (47) in [22]. In other words, the generator of the limit process is the following convex combination of $A_{i}$ :

$$
\begin{aligned}
A f=\sum_{i=0}^{2} \alpha_{i} A_{i} & =\left(\alpha_{0}+\alpha_{1}-x_{1}\right) \frac{\partial f}{\partial x_{1}}+\left(\alpha_{0}+\alpha_{2}-x_{2}\right) \frac{\partial f}{\partial x_{2}} \\
& =\left(\frac{b+x_{1}^{2}}{b+x_{1}^{2}+x_{2}^{2}}-x_{1}\right) \frac{\partial f}{\partial x_{1}}+\left(\frac{b+x_{2}^{2}}{b+x_{1}^{2}+x_{2}^{2}}-x_{2}\right) \frac{\partial f}{\partial x_{2}} .
\end{aligned}
$$

4.3. A singular perturbation in a model of fish dynamics. Paper [2] (cf. $[3,27,32])$ presents a model of dynamics of a fish population with both age and vertical structures. The fish habitat is divided into $N$ spatial patches and the fish densities $n_{i}$ in the $i$ th patch satisfy the following system of equations: 


$$
\begin{gathered}
\frac{\partial n_{i}(t, a)}{\partial t}+\frac{\partial n_{i}(t, a)}{\partial a}=-\mu_{i}(a) n_{i}(t, a)+\varepsilon^{-1} \sum_{j=1}^{N} k_{i j}(a) n_{j}(t, a) \\
n_{i}(t, 0)=\int_{0}^{\infty} b_{i}(a) n_{i}(t, a) d a, \quad i=1, \ldots, N \\
n_{i}(0, a)=\phi_{i}(a), \quad i=1, \ldots, N
\end{gathered}
$$

where $t$ stands for time, $a$ stands for age, $\phi_{i}$ is the initial population distribution in patch $i$, and $\mu_{i}$ and $b_{i}$ are age-specific and patch-specific mortality and birth rates. In the absence of the terms $\varepsilon^{-1} \sum_{j=1}^{N} k_{i j}(a) n_{j}(t, a)$ each of the patches could be treated separately, and the population densities there would satisfy the McKendrick equation [28, 31] (also called SharpeLotka-McKendrick equation [38, 39], or Lotka-McKendrick equation [20]). The matrix $k(a)=\left(k_{i j}(a)\right)$ is composed of intensities of movements between patches that occur on a daily basis: the sum of entries in each column of the matrix is zero. The factor $\varepsilon^{-1}$ (with $\varepsilon \ll 1$ ) corresponds to the fact that the age-related processes and vertical migrations (between the patches) occur at different time scales, a day being the fast time scale as compared to the fish life time.

The main question addressed in [2] is whether in modelling such populations one may disregard the vertical migration and work with a model that has been aggregated, or averaged over the whole water column. To this end, the authors assume that the matrix $k$ is irreducible, and hence possesses the unique normalized right eigenvector $\mathbf{v}(a)=\left(v_{i}(a)\right)_{i=1, \ldots, N}$ corresponding to the simple dominant eigenvalue [34]. Moreover, they consider the case where the population distribution over the patches does not change in time and agrees with the stable population distribution dictated by the matrix $k$ :

$$
\frac{n_{i}(t, a)}{n(t, a)}=v_{i}(a), \quad i=1, \ldots, N, a \geq 0,
$$

where $n=\sum_{i=1}^{N} n_{i}$. This means tacitly assuming that migrations governed by $k$ occur so fast, compared to ageing processes, that the population distribution over the patches reaches the (age-specific) equilibrium much before the ageing process intervenes; this corresponds to letting $\varepsilon \rightarrow 0$ in (16). In such a simplified, aggregated model, the population density satisfies the McKendrick equation with averaged birth and mortality rates:

$$
\begin{aligned}
\frac{\partial n(t, a)}{\partial t}+\frac{\partial n(t, a)}{\partial a} & =-\mu_{\mathrm{a}}(a) n(t, a), \\
n(t, 0) & =\int_{0}^{\infty} b_{\mathrm{a}}(a) n(t, a) d a, \\
n(0, a) & =\phi_{\mathrm{a}}(a),
\end{aligned}
$$


where "a" stands for "aggregated", $\mu_{\mathrm{a}}=\sum_{i=1}^{N} v_{i} \mu_{i}$ and $b_{\mathrm{a}}=\sum_{i=1}^{N} v_{i} b_{i}$. Here, the weights $v_{i}$ indicate the underlying, hidden spatial structure.

Equation (16) is related to a semigroup $\left\{\mathcal{T}_{\varepsilon}(t), t \geq 0\right\}$ of operators in $\mathbb{Y}$, the Cartesian product of $N$ copies of $L^{1}$ (with norm $\left\|\left(\phi_{i}\right)_{i=1, \ldots, N}\right\|_{\mathbb{Y}}=$ $\left.\sum_{i=1}^{N}\left\|\phi_{i}\right\|_{L^{1}}\right)$, where $L^{1}$ is the space of Lebesgue integrable functions on $[0, \infty)$. The generator of this semigroup is $\mathcal{A}_{\varepsilon}=\mathcal{A}+\varepsilon^{-1} \mathcal{K}$ where $\mathcal{A}\left(\phi_{i}\right)_{i=1, \ldots, N}$ $=\left(A_{i} \phi_{i}\right)_{i=1, \ldots, N}, \phi_{i} \in \mathcal{D}\left(A_{i}\right), \mathcal{K}\left(\phi_{i}\right)_{i=1, \ldots, N}=\left(\sum_{j=1}^{N} k_{i j} \phi_{j}\right)_{i=1, \ldots, N}, \phi_{i} \in L^{1}$, and $A_{i}$ is the operator in $L^{1}$ given by

$$
A_{i} \phi(a)=-\phi^{\prime}(a)-\mu_{i}(a) \phi(a)
$$

with domain $\mathcal{D}\left(A_{i}\right)$ composed of the absolutely continuous functions $\phi$ such that $\phi^{\prime} \in L^{1}$ and $\phi(0)=\int_{0}^{\infty} b_{i}(a) \phi(a) d a, i=1, \ldots, N$.

On the other hand, (17) is related to the semigroup $\{S(t), t \geq 0\}$ in $L^{1}$ generated by the operator $A_{\mathrm{a}}$ given by

$$
A_{\mathrm{a}} \phi(a)=-\phi^{\prime}(a)-\mu_{\mathrm{a}}(a) \phi(a)
$$

with domain $\mathcal{D}\left(A_{\mathrm{a}}\right)$ composed of the absolutely continuous functions $\phi$ such that $\phi^{\prime} \in L^{1}$ and $\phi(0)=\int_{0}^{\infty} b_{\mathrm{a}}(a) \phi(a) d a$.

To see that we are actually dealing with a semigroup generated by a convex combination of generators, we need to pass to the space $C=C[0, \infty]$ of continuous functions on $[0, \infty)$ with limits at infinity. To this end, we assume that the mortality and birth rates $\mu_{i}, b_{i}, i=1, \ldots, N$, belong to $C$. Then (cf. [31]) the pre-dual $A_{i, *}$ to $A_{i}$ in $C$ is given by

$$
A_{i, *} f(a)=f^{\prime}(a)-\mu_{i}(a) f(a)+b_{i}(a) f(0),
$$

and a similar statement is true for $A_{\mathrm{a}, *}$. Notably, all of these operators share the same domain $C^{1}$ composed of the differentiable functions $f \in C$ with $f^{\prime} \in C$. Moreover, $A_{\mathrm{a}, *}$ is a convex combination of $A_{i, *}$ with (age-dependent) $\alpha_{i}=v_{i}$ :

$$
A_{\mathrm{a}, *} f=\sum_{i=1}^{N} \alpha_{i} A_{i, *} f, \quad f \in C^{1} .
$$

Acknowledgments. We would like to thank J. Banasiak for drawing our attention to the paper [2], and to R. Wieczorek for drawing our attention to [33] and the literature on fast switching diffusions. This work was supported by TODEQ.

\section{References}

[1] G. K. Ackers, A. D. Johnson and M. A. Shea, Quantitative model for gene regulation by $\lambda$ phage repressor, Proc. Nat. Acad. Sci. USA 79 (1982), 1129-1133.

[2] O. Arino, E. Sánchez, R. Bravo de la Parra and P. Auger, A singular perturbation in an age-structured population model, SIAM J. Appl. Math. 60 (1999-2000), 408-436. 
[3] J. Banasiak, Asymptotic analysis of singularly perturbed dynamical systems, http:// maths.za.net/viewabstract.php?id $=109 \& \mathrm{cf}=5$.

[4] A. Bobrowski, Degenerate convergence of semigroups, Semigroup Forum 49 (1994), 303-327.

[5] -, A note on convergence of semigroups, Ann. Polon. Math. 49 (1998), 107-127.

[6] - Functional Analysis for Probability and Stochastic Processes, Cambridge Univ. Press, 2005.

[7] —, Degenerate convergence of semigroups related to a model of stochastic gene expression, Semigroup Forum 73 (2006), 345-366.

[8] - On a semigroup generated by a convex combination of two Feller generators, J. Evolution Equations 7 (2007), 555-565.

[9] —, On limitations and insufficiency of the Trotter-Kato theorem, Semigroup Forum 75 (2007), 317-336.

[10] A. Bobrowski, T. Lipniacki, K. Pichór and R. Rudnicki, Asymptotic behavior of distributions of $m R N A$ and protein levels in a model of stochastic gene expression, J. Math. Anal. Appl. 333 (2007), 753-769.

[11] A. Ciliberto, B. Novak and J. J. Tyson, Steady states and oscillations in the p53/Mdm2 network, Cell Cycle 4 (2005), 488-493.

[12] M. H. A. Davis, Piece-wise deterministic Markov processes, J. Roy. Statist. Soc. Ser. B 46 (1984), 353-388.

[13] - Lectures on Stochastic Control and Nonlinear Filtering, Springer, 1984.

[14] —, Markov Processes and Optimization, Chapman and Hall, 1993.

[15] B. Dorroh, Contraction semigroups in a function space, Pacific J. Math. 19 (1966), $35-38$.

[16] K.-J. Engel and R. Nagel, One-Parameter Semigroups for Linear Evolution Equations, Springer, New York, 2000.

[17] S. N. Ethier and T. G. Kurtz, Markov Processes. Characterization and Convergence, Wiley, New York, 1986.

[18] A. M. Femino, F. S. Fay, K. Fogarty and R. H. Singer, Visualization of single RNA transcripts in situ, Science 280 (1998), 585-590.

[19] B. Hat, P. Paszek, M. Kimmel, K. Piechór and T. Lipniacki, How the number of alleles influences gene expression, J. Statist. Phys. 128 (2007), 511-533.

[20] M. Iannelli, Mathematical Theory of Age-Structured Population Dynamics, Appl. Math. Monogr. 7, Giardini, Pisa, 1995.

[21] A. M. Il'in, R. Z. Khasminskii and G. Yin, Asymptotic expansions of solutions of integro-differential equations for transition densities of singularly perturbed switching diffusions: rapid switchings, J. Math. Anal. Appl. 238 (1999), 516-539.

[22] T. B. Kepler and T. C. Elston, Stochasticity in transcriptional regulation: origins, consequences, and mathematical representations, Biophys. J. 81 (2001), 3116-3136.

[23] T. G. Kurtz, Extensions of Trotter's operator semigroup approximation theorems, J. Funct. Anal. 3 (1969), 354-375.

[24] T. Lipniacki, P. Paszek, A. R. Brasier, B. Luxon and M. Kimmel, Mathematical model of NF-kB regulatory module, J. Theoret. Biol. 228 (2004), 195-215.

[25] - , - - - - - , Stochastic regulation in early immune response, Biophys. J. 90 (2006), 725-742.

[26] T. Lipniacki, P. Paszek, A. Marciniak-Czochra, A. R. Brasier and M. Kimmel, Transcriptional stochasticity in gene expression, J. Theoret. Biol. 238 (2006), 348367. 
[27] M. Lisi and S. Totaro, The Chapman-Enskog procedure for an age-structured population model: initial, boundary and corner layer corrections, Math. Biosci. 196 (2005), 153-186.

[28] J. D. Murray, Mathematical Biology, 2nd ed., Springer, 1993.

[29] P. Paszek, Modeling stochasticity in gene regulation: characterization in the terms of the underlying distribution function, Bull. Math. Biology 69 (2007), 1567-1601.

[30] L. C. G. Rogers and D. Williams, Diffusions, Markov Processes and Martingales, Vol. 1, Foundations, Cambridge Univ. Press, 2000.

[31] R. Rudnicki, Population Dynamics, unpublished lecture notes (in Polish).

[32] E. Sánchez, R. Bravo de la Parra, P. Auger and P. Gómez-Mourelo, Time scales in linear delayed differential equations, J. Math. Anal. Appl. 323 (2006), 680-699.

[33] V. V. Sarafyan and A. V. Skorohod, On fast switching dynamical systems, Theory Probab. Appl. 32 (1987), 595-607.

[34] E. Seneta, Non-Negative Matrices and Markov Chains, 2nd ed., Springer, 1981.

[35] M. A. Shea and G. K. Ackers, The Or control system of bacteriophage lambda: a physical-chemical model for gene regulation, J. Molecular Biol. 181 (1985), 211-230.

[36] M. Sova, Convergence d'opérations linéaires non bornées, Rev. Roumaine Math. Pures Appl. 12 (1967), 373-389.

[37] M. Thattai and A. van Oudenaarden, Intrinsic noise in gene regulatory networks, Proc. Nat. Acad. Sci. USA 98 (2001), 8614-8619.

[38] G. F. Webb, Theory of Nonlinear Age-Dependent Population Dynamics, Dekker, 1985.

[39] —, Structured population dynamics, in: Mathematical Modelling of Population Dynamics, R. Rudnicki (ed.), Banach Center Publ. 63, Inst. Math., Polish Acad. Sci., 2004, 123-163.

[40] G. Yin, On limit results for a class of singularly perturbed switching diffusions, J. Theoret. Probab. 14 (2001), 673-697.

[41] G. Yin and M. Kniazeva, Singularly perturbed multidimensional switching diffusions with fast and slow switchings, J. Math. Anal. Appl. 229 (1999), 605-630.

Institute of Mathematics

Polish Academy of Sciences

Katowice branch

Bankowa 14

40-007 Katowice, Poland

on leave from

Department of Mathematics

Faculty of Electrical Engineering

and Computer Science

Lublin University of Technology

Nadbystrzycka 38A

20-618 Lublin, Poland

E-mail: a.bobrowski@pollub.pl
Actuarial and Insurance Solutions

Deloitte Advisory Ltd.

Piękna 18

00-549 Warszawa, Poland

E-mail: rbogucki@deloittece.com

Received January 11, 2008

Revised version October 8, 2008 